\title{
Presentación
}

\section{Conmemoración de la Conferencia Mundial de Salamanca y su Influencia en América Latina}

\author{
Commemoration of the Salamanca World Conference and its \\ influence in Latin America
}

\author{
Rosa Blanco * \\ Cynthia Duk
}

Universidad Central de Chile, Chile

Este monográfico está dedicado a la conmemoración de los 25 años de la Conferencia Mundial sobre Necesidades Educativas Especiales: acceso y calidad, celebrada en Salamanca, España, del 7 al 10 de junio de 1994, y organizada por la UNESCO y el Gobierno de España. Esta Conferencia constituyó un compromiso internacional de gran trascendencia para avanzar en el cumplimiento del derecho a la educación, particularmente de los estudiantes con necesidades educativas especiales y otros en situación de exclusión, sentando las bases para el desarrollo de escuelas y sistemas educativos inclusivos, que se han ido reforzando y profundizando en compromisos internacionales posteriores. Junto con celebrar el aniversario de la Conferencia, se da cuenta de su contribución e influencia en América Latina, desde la perspectiva de diferentes actores educativos de cinco países de la región.

En Salamanca se dieron cita representantes de alto nivel de 92 gobiernos y de 25 organismos internacionales y ONGS, así como especialistas, representantes de colectivos docentes y profesionales con el propósito de reafirmar su compromiso de la Educación para Todos, representando un nuevo punto de partida para hacer efectivo su derecho a educarse en las escuelas de su comunidad, es decir, a la escuela que debiera acudir si no tuviera ninguna discapacidad" (Artículo 18, UNESCO, 1994).

La Conferencia se centró en dos grandes preocupaciones: Garantizar a todos los niños, en particular con necesidades educativas especiales, el acceso a las oportunidades educativas y; actuar para conseguir que sean de calidad. La reflexión y los debates se organizaron en torno a cuatro temas; políticas y legislación; factores escolares; perspectivas comunitarias, asociaciones y redes. Los compromisos adoptados por todos los participantes, como resultado de los trabajos realizados, quedaron expresados en la Declaración y Marco de Acción para las Necesidades Educativas Especiales, estableciendo un conjunto de principios, disposiciones y recomendaciones para guiar la acción de los gobiernos, organizaciones de la sociedad civil y organismos internacionales.

Salamanca marcó sin duda un hito en materia de educación inclusiva por varias razones. En primer lugar, porque dio visibilidad y puso en el primer plano a uno de los grupos sociales más excluidos y segregados de la educación, y con frecuencia ausentes en las políticas y reformas educativas. De esta manera se dio continuidad a los compromisos

*Contacto: rblanco.guijarro@gmail.com 
adoptados en la Conferencia Mundial sobre Educación para Todos: Satisfacción de las Necesidades Básicas de Aprendizaje, Jomtien 1990, donde una de las recomendaciones fue "universalizar el acceso a la educación y fomentar la equidad, tomando medidas sistemáticas para reducir las desigualdades y suprimir las discriminaciones referidas a las posibilidades de aprendizaje de los grupos más desasistidos, prestando especial atención a las necesidades de aprendizaje de las personas impedidas y tomar medidas para garantizar a estas personas la igualdad de acceso a la educación como parte integrante del sistema educativo (Artículo 3, UNESCO, 1990).

Significó también una oportunidad para reforzar el artículo 6 de las Normas Uniformes sobre la igualdad de Oportunidades para las Personas con Discapacidad, aprobadas por la Asamblea de Naciones Unidas el 20 de diciembre de 1993, donde se expresa que "los Estados deben reconocer el principio de la igualdad de oportunidades de educación en los niveles primario, secundario y superior para los niños, los jóvenes y los adultos con discapacidad en entornos integrados, recibiendo los apoyos apropiados y velando porque la educación de las personas con discapacidad sea una parte integrante del sistema de enseñanza" (ONU, 1993, p.16).

Aunque el foco de Salamanca fueron las necesidades educativas especiales, los principios y propuestas de su marco de Acción constituyeron un primer compromiso internacional para avanzar hacia el desarrollo de escuelas inclusivas que acojan y reconozcan la diversidad del alumnado y transformen su organización, cultura y prácticas para favorecer la participación y el aprendizaje de todos. Para lograr este propósito, otro gran mensaje de la Conferencia fue la necesidad de transformar los sistemas educativos -curriculum, evaluación, formación docente, entre otros-, así como la educación y servicios de educación especial para apoyar los procesos de inclusión.

Los principios y propuestas de Salamanca han tenido una influencia significativa en los países de la región, aunque persisten numerosas barreras y desafíos, tal como lo expresan los diferentes autores de este monográfico, para que todas las escuelas, y no solo parte de ellas se comprometan con la inclusión. Una posible explicación es que la Conferencia tuvo lugar en un escenario regional de entusiasmo y transformaciones significativas, que situaban la educación como elemento central para el desarrollo con equidad y el fortalecimiento de las democracias, incipientes en muchos países tras un periodo de dictaduras.

En la década de los noventa se inician reformas educativas, acompañadas de grandes inversiones, que, sin descuidar el aumento de la cobertura, se centran fundamentalmente en la transformación curricular y la gestión de los sistemas educativos para lograr una educación de calidad con equidad, asegurando aprendizajes de igual calidad para toda la población escolar, especialmente para la que se encuentra en situación de mayor vulnerabilidad (OREALC/UNESCO, 2001, p. 53). Este escenario constituyó un terreno propicio para adoptar los principios y propuestas de Salamanca, especialmente en una región caracterizada por grandes desigualdades educativas y sociales, y una acentuada fragmentación social, cultural y educativa.

Junto con esta situación favorable al cambio educativo en los países, otro factor que influyó de manera importante fue el rol de la UNESCO, y particularmente de la Oficina Regional de Educación para América Latina y el Caribe, OREALC/UnESCO Santiago, en el impulso y seguimiento de la Agenda de Salamanca y de otros compromisos internacionales posteriores. Una de las principales acciones de la UNESCO ha sido posicionar la educación 
inclusiva en la agenda de las políticas educativas, mediante la realización de foros técnicopolíticos con ministros de educación y otros responsables de las políticas educativas, fomentando el diálogo, la promoción de ideas y el intercambio entre los países.

En este sentido, resulta relevante el compromiso adoptado en la VI Reunión de Ministros de Educación de América Latina y el Caribe, celebrada en Kingston, Jamaica, 1996, donde por primera vez una de las recomendaciones hace referencia al principio rector de Salamanca al establecer la necesidad de "Fortalecer las condiciones y estrategias que favorecen el desarrollo de las escuelas para que atiendan niños con necesidades especiales o que presentan dificultades de aprendizaje debidas a diferentes causas, tales como: discapacidades físicas, enseñanza o escolaridad inadecuada y ambientes sociales marginados. Las escuelas integradoras favorecen la igualdad de oportunidades, proporcionan una educación más personalizada, fomentan la solidaridad y cooperación entre los alumnos y mejoran la calidad de la enseñanza y la eficacia de todo el sistema educativo" (OREALC/UNESCO, 1996).

Una década después se dio otro paso importante en la Segunda Reunión del Comité Intergubernamental del Proyecto Regional de Educación para América Latina y el Caribe "PRELAC II"-, que tuvo lugar en 2007 en Buenos Aires, Argentina. En ella se debatió el documento base "Educación de calidad para Todos: un asunto de derechos humanos" en el que se considera la educación como un bien público y un derecho del que nadie puede estar excluido, definiendo la calidad de la educación a través de cinco dimensiones: equidad, relevancia, pertinencia, eficacia y eficiencia (OREALC/UNESCO, 2007). En la Declaración de Buenos Aires se establece que "es urgente avanzar hacia sistemas y centros educativos más inclusivos, formulando estrategias que contribuyan a romper el circuito de reproducción de la pobreza y la exclusión social" (OREALC/UNESCO, 2007). En este contexto, los ministros de educación de comprometieron a desarrollar políticas inclusivas con miras a la mejora de la calidad y la pertinencia de la educación que incidan en todos los actores del sistema educativo.

Junto con la realización de los foros técnico-políticos, la Oficina Regional de Educación de la UNESCO, ha prestado asistencia técnica para la formulación de normativas, políticas y planes, así como para el desarrollo de experiencias locales de inclusión, con la finalidad de apoyar a los países adquirir la experiencia necesaria para su expansión y generalización, que fue una de las recomendaciones de Salamanca. Otra acción de especial relevancia ha sido el fortalecimiento de capacidades de los responsables y gestores de políticas, docentes, formadores de docentes y sociedad civil, así como la producción y difusión de documentos y materiales de formación para apoyar el desarrollo de políticas y prácticas inclusivas.

Con la Conferencia de Salamanca la UNESCO puso en marcha el motor para avanzar hacia sistemas educativos y escuelas más inclusivas, y ha seguido impulsando la agenda internacional en esta dirección, desarrollando nuevas perspectivas que han favorecido el avance del pensamiento y el desarrollo de políticas y prácticas. En este sentido cabe destacar la Conferencia Internacional Educación Inclusiva: el camino hacia el futuro, organizada por la Oficina Internacional de Educación de la UNESCO en 2008, donde se amplía la perspectiva de la inclusión, concibiéndola como un proceso permanente cuyo objetivo es ofrecer una educación de calidad para todos, respetando la diversidad de los educandos y de las comunidades y eliminando toda forma de discriminación.

El impulso más reciente se produce con la Agenda Mundial de Educación 2030 para avanzar hacia el ODS4: "Garantizar una educación inclusiva y equitativa de calidad, y 
promover oportunidades de aprendizaje a lo largo de la vida para todos". Desde esta perspectiva la equidad y la inclusión están en la base de una educación de calidad y ninguna meta educativa debería considerarse lograda a menos que se haya logrado para todos (UNESCO, 2015).

La relevancia y el legado de la Conferencia de Salamanca ameritan un número monográfico en su 25 aniversario, como un reconocimiento de su contribución al desarrollo de una educación inclusiva de calidad, que es la preocupación central de esta revista. Los artículos temáticos abordan los principales temas y propuestas de la Conferencia, dando cuenta de las perspectivas y recorridos realizados en algunos países de América Latina en los distintos ámbitos. Desde la situación en el campo de la educación inclusiva, pasando por el desarrollo de políticas, la transformación de la educación especial, los sistemas de apoyo, la formación inicial de los docentes, el rol de las asociaciones de las personas con discapacidad y sus familias, y la inclusión en Educación Superior.

En el primer artículo, Rosa Blanco y Cynthia Duk, desarrollan un análisis y reflexión de lo que ha significado la Conferencia de Salamanca, desde las voces de ocho participantes en la misma, incluidas las autoras. Se destacan sus principales mensajes y contribuciones en el desarrollo del pensamiento, políticas y prácticas inclusivas y su influencia en los países de América Latina, concluyendo con las principales amenazas y los desafíos para avanzar hacia escuelas y sistemas educativos inclusivos.

En siguiente trabajo, Álvaro Marchesi y Laura Hernández analizan los avances y los desafíos sociales y educativos en los países latinoamericanos, destacando los temas emergentes del del siglo XXI, pero sin olvidar la agenda pendiente del siglo XX. Desarrollan cinco prioridades políticas, sociales y educativas que impulsarían la inclusión social y educativa: los acuerdos políticos, la prioridad de la infancia, el fortalecimiento de la profesión docente, la transformación cultural y el cambio en las actitudes.

El artículo de Ricardo Rosas, James Staig, Guillermo Lazcano, Rosario Palacios, Mikko Aro y Calendaria Ibernon, ofrece un análisis comparado de los sistemas de Educación Especial de Chile, España y Finlandia, desde la perspectiva de la Declaración de Salamanca. Se examinan varias dimensiones: contexto general de los sistemas educativos, orientación epistemológica de la Educación Especial, marcos regulatorios, procedimientos de detección y derivación de niños con NEE, la estructura de los servicios de atención a la diversidad y las estrategias de inclusión de los estudiantes con NEE.

Rodolfo Cruz analiza las implicaciones de tuvo en México la Declaración de Salamanca, desde la transformación de la educación especial y el desarrollo de políticas de Integración Educativa, hasta llegar a los principios de una escuela inclusiva de la actual política. El autor plantea cinco preguntas complejas en torno a los retos, tensiones y disputas sobre la puesta en marcha y concreción de los ideales inclusivos, con objeto de favorecer la reflexión sobre su implementación desde una perspectiva situada, que considere las distintas realidades de los países.

El artículo de Cynthia Duk, Tatiana Cisternas y Liliana Ramos aborda el tema de la formación inicial docente desde un enfoque inclusivo, analizando la influencia de los cambios propuestos en Salamanca en relación con las escuelas y docentes, así como su presencia en las políticas educativas de Chile. Se revisan tres propuestas sobre las capacidades que debe promover la formación inicial docente para encarnar los principios de la inclusión educativa, proponiendo, a partir de dicho análisis, cuatro competencias 
fundamentales para la formación de todos los profesores y algunas recomendaciones para las políticas educativas.

El artículo de Catalina Andujar, aborda un tema clave para la inclusión, el apoyo a los aprendizajes para que ningún estudiante se quede atrás, destacando los aportes de Salamanca en este ámbito. Aborda las condiciones para el desarrollo de escuelas inclusivas con énfasis en el apoyo a los aprendizajes, como denominador común de los mejores sistemas educativos del mundo. Presenta algunas experiencias y políticas de apoyo de República Dominicana, concluyendo con una reflexión sobre los riesgos de una visión limitada de los apoyos y la necesidad de desarrollar marcos y políticas integrales de apoyo.

Ismael García y Silvia Romero, analizan la influencia de la Declaración de Salamanca en la atención a la diversidad en México, haciendo un breve recorrido de los principales cambios en la organización y legislación de la educación desde la década de los noventa, así como los problemas que han ido surgiendo en relación con el papel que la Educación Especial debe jugar dentro del movimiento de Educación Inclusiva. Argumentan que, si bien la Educación Especial requiere una transformación profunda no debe desaparecer, pues es parte esencial del tránsito hacia la Educación Inclusiva.

María Eugenia Yaradola hace una reflexión sobre el aporte de la Conferencia de Salamanca a las Organizaciones no Gubernamentales de y para las personas con discapacidad y sus familias, en la defensa por una inclusión educativa. Analiza también algunas de las fisuras del Marco de Acción que han limitado un avance más decido hacia una educación inclusiva con ejemplos de las políticas y prácticas de Argentina. Finaliza con una serie de desafíos para que las ONGS/PCDF sean realmente inclusivas e incidan en la construcción de sistemas educativos y sociedades más inclusivas.

La Educación Superior fue poco tratada en la Conferencia de Salamanca, pero actualmente es uno de los desafíos emergentes en la Región, como consecuencia de los procesos de inclusión en niveles anteriores. Sonia Brito y Lorena Basualto, problematizan la inclusión en Educación Superior, desde el desarrollo del binomio social y educativo, como una forma de enfrentar la educación desde una arista social, donde es considerada como un derecho humano y, por tanto, intrínsecamente inclusiva. Parten del supuesto que la educación es un quehacer esencial del progreso de la humanidad que posibilita el desarrollo de las personas y potencia sus capacidades en aspectos morales, sociales y culturales.

Rafael Bell, Belinda Lema, Emma Zulay y Alejandro Lema, hacen una reflexión acerca de la comprensión y el carácter de la educación superior inclusiva, en lo referido a las personas con discapacidad, y su desarrollo en el Instituto Superior Tecnológico de Formación Profesional, Administrativa y Comercial (ITF) de Guayaquil, Ecuador, como expresión de la respuesta a la necesidad de avanzar hacia el itinerario delineado en Salamanca. Basándose en una investigación realizada en dicha institución aportan elementos sobre su recorrido y etapas en materia de inclusión.

La sección de trabajos libres consta de cinco artículos. El primer artículo, cuya autora es Paola Andreucci, trata el tema de la atención temprana, que fue una de las prioridades del Marco de Acción de Salamanca. Aborda la discapacidad intelectual desde una perspectiva sistémico-familiar, relevando el aporte de los distintos miembros en la construcción del fenómeno, dando la voz a los/as hermanos/as de niños/as con síndrome de Down, indagando en sus representaciones respecto de sí mismos, sus hermanos/as en situación de discapacidad y su rol en la familia. 
Gabriela Naranjo, analiza cómo se asumen en las aulas los desafíos de la inclusión en la actual política educativa mexicana, a partir de registros etnográficos de la práctica de cinco maestros en escuelas primarias de la Ciudad de México. Las etnografías consideran las perspectivas de los docentes sobre la diversidad de sus alumnos, los desafíos que plantea a su trabajo cotidiano y las formas en que los afrontan. Enfatiza la necesidad de complejizar la mirada sobre el trabajo docente y generar las condiciones necesarias para hacer viable en las aulas una educación inclusiva.

Eduardo Llanos desarrolla un análisis de la película francesa "Los coristas” (Les choristes), que muestra un caso de intervención e innovación pedagógica con niños en riesgo social. Examina seis dimensiones del filme (interpersonal, intrapersonal, transpersonal, ideológica, simbólica y estética), haciendo un análisis mediante seis niveles de comprensión y apreciación: descriptivo, inferencial, interpretativo, valorativo, introspectivo y heurístico o creativo.

Violeta Quiroz, Melissa Sennas y Sylvia Contreras, abordan las representaciones de género en los profesores noveles de educación general Básica de Chile, desde el marco de la educación inclusiva, mediante la realización de un estudio cualitativo descriptivo. Entre los hallazgos, aprecian una transición en la categoría de género asociado al biologismo y también a lo sociocultural, observándose inconsistencias y contradicciones con sus discursos respecto a la Educación Inclusiva.

El último artículo de Francisco Javier Gil-Llambías, Rodrigo del Valley, Mirza Villarroel y Carolina Fuentes, se enmarca en las políticas de acceso inclusivo a la Educación Superior en Chile, a través del Programa de Acompañamiento y Acceso Efectivo a la Educación Superior (PACE). Se ofrece una caracterización de estudiantes de la cohorte 2017 de este programa en comparación con estudiantes de un Grupo Control y, se investiga su desempeño académico en las universidades Católica del Norte, de Santiago de Chile y Católica de Temuco.

El presente monográfico puede ser leído como un patrimonio del camino recorrido por los países de América Latina desde Salamanca para garantizar a toda la ciudadanía, y particularmente a quienes están excluidos o segregados, el derecho a una educación equitativa e inclusiva de calidad, que respete y valore la diversidad humana y contribuya al desarrollo de sociedades más justas, inclusivas y democráticas. Queda mucho por recorrer, pero, como expresan diversos autores el camino iniciado en Salamanca no tiene retorno porque la mayoría de sus mensajes siguen vigentes e inconclusos.

\section{Referencias}

OREALC/UNESCO. (1996). Declaración VI Reunión de Ministros de Educación de América Latina y el Caribe. Kingston, Jamaica. Santiago de Chile: UNESCO.

OREAlC/UnESCO. (2001). Balance de los 20 años del Proyecto Principal de Educación en América Latina y el Caribe. Santiago de Chile: UNESCO.

OREALC/UNESCO. (2007). Educación de calidad para Todos: Un asunto de Derechos Humanos. II Reunión del Comité Intergubernamental del Proyecto Regional de Educación para América Latina y el Caribe EPT/PRELAC. Santiago de Chile: UNESCO.

OREALC/UNESCO. (2007). Declaración de Buenos Aires. Reunión Regional de Ministros de Educación de América Latina y el Caribe. Buenos Aires. Santiago de Chile: UNESCO 
Organización de Naciones Unidas. (1993). Normas uniformes sobre la igualdad de oportunidades para las personas con discapacidad. Nueva York, NY: ONU.

UNESCO. (1990). Declaración Mundial sobre Educación para Todos. Satisfacción de las necesidades básicas de aprendizaje. Jomtien, Tailandia. París: UNESCO.

UNESCO. (1994). Informe final de la Conferencia Mundial sobre Necesidades Educativas Especiales: Acceso y Calidad. Salamanca, España. París: UNESCO.

UNESCO. (2015). Declaración de Incheon y Marco de Acción para la realización del Objetivo de Desarrollo Sostenible 4: Garantizar una educación inclusiva y equitativa de calidad y promover oportunidades de aprendizaje permanente para todos. París: UNESCO.

\section{Breve CV de las autoras}

\section{Rosa Blanco}

Directora de la Organización de Estados Iberoamericanos en Chile entre 2012 y 2018. Especialista regional en educación inclusiva y educación de la Primera Infancia de la Oficina Regional de Educación de UNESCO para América Latina y el Caribe, entre 1994 y 2011, ejerciendo la dirección interina entre 2007 y 2008. Asesora técnica del Centro Nacional de Recursos para la Educación Especial y del Centro de Desarrollo Curricular del Ministerio de Educación de España entre 1986 y 1994. En la actualidad presta asesoría y docencia en el área de Formación Continua y de Investigación del Centro de Desarrollo e Innovación en Educación Inclusiva de la Universidad Central de Chile, además es profesora visitante del Postgrado de Especialización en Inclusión Educativa de Personas con Discapacidad, e investigadora en el parea de inclusión de Personas con Discapacidad: de la Universidad Católica de Córdoba, Argentina. Ha realizado diversos estudios y numerosas publicaciones sobre educación inclusiva, educación de la primera infancia, calidad y equidad de la educación. ORCID ID: https://orcid.org/0000-0001-6263-3004. Email: rblanco.guijarro@gmail.com

\section{Cynthia Duk}

Directora del Centro de Desarrollo e Innovación en Educación Inclusiva y del programa de Magíster en Educación Inclusiva de la Universidad Central de Chile. Además, es Directora/editora de la Revista Latinoamericana de Educación Inclusiva que publica la Facultad de Educación en colaboración con la RINACE. Red Iberoamericana de Investigación sobre Cambio y Eficacia Escolar. Su línea principal de desarrollo e investigación es la inclusión y la diversidad en educación, con énfasis en el desarrollo de escuelas inclusivas. Lideró un Proyecto I+D de Conicyt, fruto del cual se construyó el Modelo INCLUSIVA para evaluar y mejorar la respuesta de la escuela a la diversidad; así como el Estudio del Mineduc "Criterios y orientaciones para flexibilizar el currículo en los distintos niveles de enseñanza”. Tiene varias publicaciones y ha diseñado numerosos programas y materiales de formación y desarrollo profesional, destaca el material de formación docente Educar en la Diversidad para los países del MERCOSUR y el Diplomado e-learning de la OEI, "Escuelas Inclusivas: enseñar y aprender en la diversidad" para Iberoamérica. ORCID ID: https://orcid.org/0000-0002-8443-0707. Email: cduk@ucentral.cl 